\title{
Contents, Vol. 111, 1946
}

\section{Index}

Amsler, M., Kératocône classique et kératocône fruste ; arguments uni-

taires 96

Discussion : Rintelen.

- $\quad$ und Huber, A., Methodik und erst $>$ e Ergebnisse einer Funktionsprü-

fung der BÍut-Kammerwasser-Schranke. (Hierzu Tafel III) . 155 Diskussion: Goldmann, Bruckner.

- $\quad$ et Verreg, F., Hétérochromie de Fuchs et fragilité vasculaire . . 177André-van Leeuwen, M., Aspects oliniques et problème du terrain de la

pupillotonie. (Recherches familiales - Note prélimin < aire) . . 339 Aschmann, A., vide

Goldmann, H. Babel, J., et Campos, R., Sur la regeneration des nerfs dans les greffons

oornéens $\quad 140$

Balavoine, C, vide Franceschetti, A.

Bangerter, A., Behandlung der Amblyopie 220

- Zur Schielbehandlung 221

Diskussion: Haemmerli, Goldmann.

Barraquer Moner, J,-L, Écarteur pour ГOpération du Décollement de la

Rétine 61

Birkhäuser, R., vide Delia Casa, F.

Bischler, V., Dystrophie cornéenne nodulaire de Salzmann . . Ill

Blum, John-D., vide Valerio, M.

Bourquin, J. B., Conjonctivite de Parinaud et Syndrome de Löffler . 73

Bienta, J., et Danis, P., L'hémianopsie bitemporale d'origine traumatique 8

Bruckner, R., Über Histaminwirkung am Auge 306

Bürki, E., Zur Kenntnis der erblichen Epitheidystrophie der Hornhaut

(Typus Meesmann): Ein weiterer Stammbaum 134

Campos, R., vide Babel, J.

Danis, P., vide Brenta, J.

Delia Casa, F., Metastatische Abszeßbilding in der Vorderkammer des

Auges bei chronischer Osteomyelitis. (Hierzu Tafel II) ... 152

- $\quad$ und Birkhäuser, R., Zur offiziellen Einführun $3 / 4$ der Dunkeladapta-tionsprüfung im

Verkehrswesen 315

Dufour) R.t Repérage aux rayons X des corps étrangers ocula $1 / 8 \mathrm{ses}$. Un

nouveau graphique 310

Ferrié, J., Importance de $\Gamma$ lnterrogatoire des malades pour le diagnostic

de la tuberculose oculaire $\quad 332$

Franceschetti, A., Les affections oculaires d'origine rhumatismale . . 242 Discussion : Rintelen, Bruckner, Goldmann. --· Sarasin, R., et Balavoíne, C, Les rayons Bucky ou rayons limites en ophtalmologie 302 
Goldmann, H., Demonstration unseres neuen Projeklionskugelperimeters samt theoretischen und klinischen Bemerkungen über Perimetrie . 187

- Abfluß des Kammerwassers beim Menschen 146

Diskussion: Bruckner.

- $\quad$ und Aschmann, A., Studien über Akkommodation (über Korrekturvon

Akkommodationsparese sowie über «physikalische und physio-

logische Akkommodationsbreite») 182

Diskussion: Franceschetti.

Berichtigung

Gruber, M., «Cornea verticillata». Eine einfach-dominante Variante der

Hornhaut des menschlichen Auges. (Hierzu Tafel I.) - 1. Mitteihmg 120 Diskussion: Goldmann.

Huber, A., vide Amsler, M.

Kenel, Ch., Les sangsues, Thirudine et leurs princípales applications en ophtalmologie 299

Knapp, P., Einseitige Alopecie der Augenbraue $\quad 67$

- $\quad$ und Schwarzmann, A., Beitrag zur Frage diencephal bedingter Ge-

sichtsfeldeinschränkung 270

Knüsel, 0., Neues über Kontaktschalen 291

Doppelfärbung Scharlachrot/Methylenblau. Klinischer Wert einer Doppelfärbung der

Conjunctiva in vivo ...... 295

Demonstration von Gesichtsfeldschemata einer Arachnoiditis optica-chiasmatica 298

- $\quad$ Morphologie einer Cataracta electrica 298

Maeder, G., Héméralopie et syndactylie familiales 278

Meyer-Hartmanni W. M., Bäderbehandlung der rheumatisehen Erkran-

kungen unter besonderer Berücksichtigung der Thermen von Baden 229 Rintelen, F., Penicillin-

Therapie beim Ulcus serpens .... 102 Diskussion: Streiff, Saubermann.

- Zur Ätiologie und Therapie der sogenannten Keratoconjunctivitisepidemica

108

- $\quad$ Über arteriosklerotische Opticusatrophie 285

Rønne, G., Penicillin Treatment of "Late Infection” Following Fistulating

Operations 1

Sarasin, R., vide Franeeschetti, A.

Schmidt, A. E., Dysmorpho-dystrophia mesodermalisi congenita (Weve,

Marfan, Marchesani). Analyse und Synthese ..... 28

Über Lichtreflexe bei Sphärophakie. (Hierzu Tafel IV) . . . 359

Beitrag zur Kenntnis des Kontusions- und Massagestares: Definitive

und flüchtige Formen 365

Schwarzmann, A., vide Knapp, P.

Streiff, E. B., Angio-tГopho-neurose généralisée et glaucoma ... 68

- $\quad$ et Zwahlen, P., Une famille avec dégénérescence en bandelette de lacornée

Valerio, M., et Blum, John-D., Reclierches sur Гaction mydriatique de

I'adrénaline $\bullet$ en instillations. Influence des substances mouillantes 193

Velden, H. A. van der, The Number of Quanta Necessary for the Perception of Light of the

Human Eye 321

Vethage, J. W. C, Chorio-Rétinite juxtapapillaire (Jensen) . . . 351

Verrey, F., Signification pratique de la phagocytose intra-oculaire . . 222 Discussion : Bruckner.

- $\quad$ vide Amsler, M.Zwahlen, P., vide Sfreí//, E.B. 
GESELLSCHAFTSBERICHTE - SOCIETY TRANSACTIONS - SOCIfiTÉS

S. Schweizerische Ophthalmologische Gesellschaft - Société Suisse d'Oph-talmologie. 38. Generalversammlung in Baden

6. und 7. Oktober 1945 - 38e Assemblée générale à Baden

6 et 7 octobre $1945 \ldots 65$

On Adaptation: An Apology (Letter to the Editor) 372 